\title{
EXAggerated CARToON STYLE Motion IN HOTEL TRANSYLVANIA
}

\author{
Chris Carter \\ Animation, Queensland University of Technology, Brisbane, Australia
}

\begin{abstract}
The fundamental principles of animation were developed at the Disney studio in the 1930s and when applied to animation result in a recognisable "Disney" style of motion. By adhering to these fundamental principles of animation, Animators have been able to successfully extendthe Disney style of motion to the 3D CG medium. This papergoes beyond Disney style motion andexamines the adaptation of techniques typically seen in more extreme cartoon-style motion and "pushed posing", exemplified by early Warner Brothers animated short films. This is achieved via an analysis of Hotel Transylvania by Sony Pictures Animation, which features a stylised form of animation adapted from the extended principles of extreme cartoon-style motion to the $3 D$ medium.
\end{abstract}

\section{KEYWORDS}

Character Animation, Motion Style, Cartoon Style, Hotel Transylvania, 3D Animation

\section{INTRODUCTION}

Research and development at the Walt Disney Studios conducted in the 1930s resulted in the development of a naturalistic cartoon style of motion, which quickly became the dominant aesthetic of animated feature films. Twelve fundamental principles of Disney animation were developed and taught to new animators "as if they were the rules of the trade" [1, p. 47]. Through continued research and development, Disney animation eventually reached a point where the principles became so refined that it progressively became harder for animators to identify each one. However, outside of the Disney studio, others such as Warner Brothers (WB) and MetroGoldwyn-Mayer (MGM) began applying the principles in vivid displays of broad character action. Some, such as the creator of The Ren and Stimpy Show, John Kricfalusi[2], go as far to suggest that WB and MGM developed a style of animation that had a better application of the principles than the Disney animators who discovered them. Other studios such as United Productions America (UPA) took animation in a different direction by focussing more on graphic symbolism and a limited movement vocabulary. While in Japan, manga artist Tezuka Osamu developed a style of animation that was inspired by Disney yet significantly different in its approach to motion and staging. Possibly due to a lack of understanding on how to produce animation and drawing upon his success as a manga artist, Tezuka's early works combined a more cinematic layout with a limited movement vocabulary [3, p.99]. This quickly inspired a form of animation so abundant in Japan and recognisable as a unique style that the truncated term Anime was adopted to differentiate it from other forms of cel-based animation. These early forms of animation clearly had stylistic qualities that separated them, 
however, since then 3D CG animation has replaced cel-animation as the dominant form. As this paper demonstrates via an analysis of Hotel Transylvania[4], the range of movement styles in 3D animation has continued to expand as artists trained in traditional techniques adapt these early movement styles to the new medium.

As my previous analysis of the character motion in Tangled[5] has shown, the Disney studio has continued to extended traditional Disney aesthetics into computer animation [6]. Other studios however, such as DreamWorks, BlueSky and Sony Pictures Animation tend to use a broader and more liberal application of the principles closer to the WB and MGM movement vocabulary. Hotel Transylvania is an animated feature film created by Sony Pictures Animation in which the director, GenndyTartakovsky, has deliberately adapted the styles of WB and MGM into the CG medium. This paper looks closely at the character animation in Hotel Transylvania to understand how an exaggerated movement style has been achieved in the film under Tartakovsky's guidance.

\section{Production Context}

The visual style of Hotel Transylvania owes a lot to the creative vision of its director GenndyTartakovsky, who is well known for his distinct visual style in the 2D medium. Hotel Transylvania is Tartakovsky's first animated feature film and his first 3D CG animated film, however, he has directed and produced a large number of stylised 2D animated series for Cartoon Network including Power Puff Girls; Dexter's Laboratory; Samurai Jack; Star Wars: Clone Wars; as well as Sym-Bionic Titan [7]. Unlike the Disney naturalistic style of animation, Tartakovsky's earlier works feature a flat, graphic angular styling with strong character posing and sharp exaggerated movement patterns. The following images from Samurai Jack and Star Wars: Clone Wars demonstrate this distinct visual style:

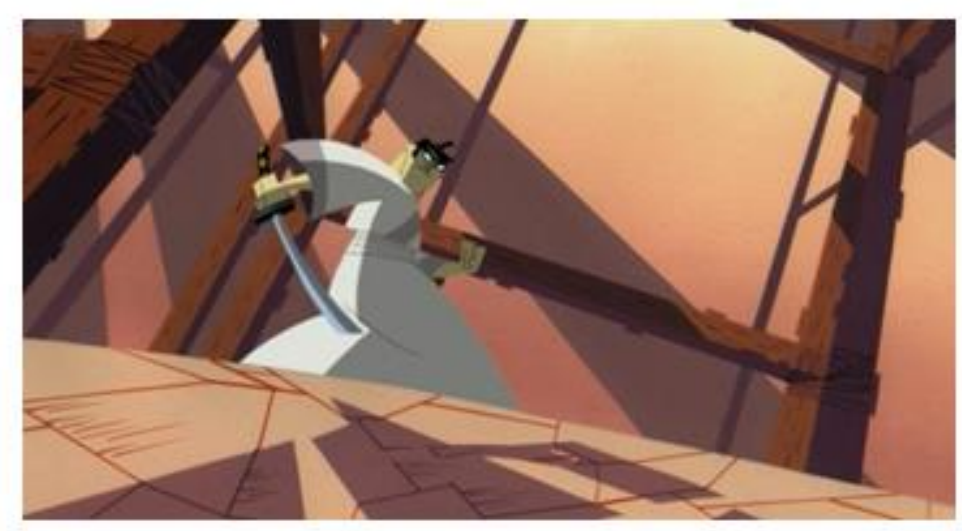

Figure 1. Graphic Stylisation in Samurai Jack. 


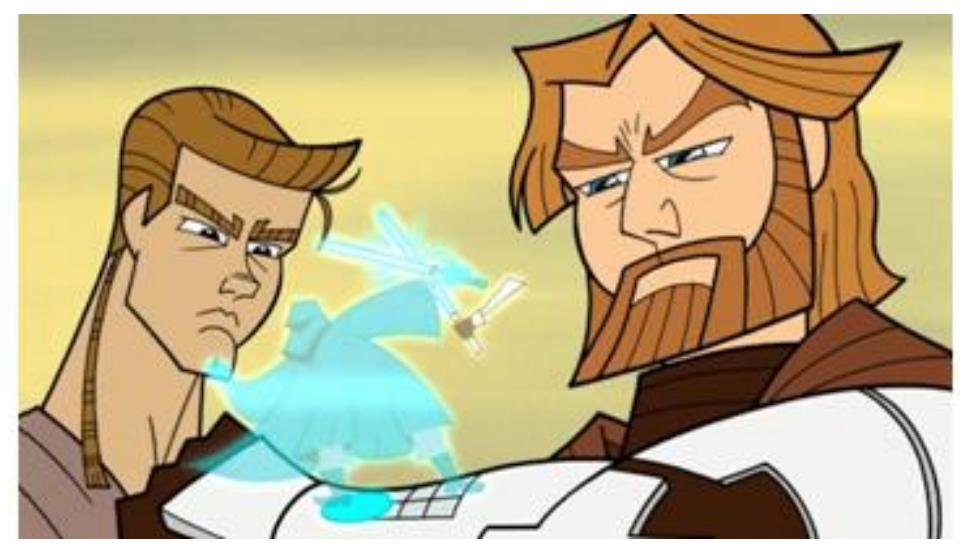

Figure 2. Graphic Stylisation in Star Wars: Clone Wars.

The visual style of Samurai Jack and Star Wars: Clone Wars draw inspiration from the graphic symbolism that UPA are known for with the cinematic feel of Japanese anime. In terms of movement the characters tend to hold extreme dynamic poses and transition quickly from one pose to the next. Recognising the visual style of Tartakovsky's earlier works is significant to understanding his approach in the 3D medium.

Studios such as PIXAR and Disney tend to retain a consistent movement and visual style across their films, otherwise known as a "house style". Michelle Murdocca, producer at Sony Pictures Animation (SPA) claims that SPA prides itself on not imposing any particular house style on its animated films:

We give our directors the latitude to bring with them the style of the movie they're creating. That's what makes the movies we've made here different and distinctive - they don't follow any given pattern. [8, p. 14]

With the freedom to explore new styles, Tartakovsky has used his 2D animation sensibilities to develop a movement vocabulary in Hotel Transylvania that differs from typical Disney style motion as well as previous SPA films. As Murdocca reinforces, "Hotel Transylvania has a very different look and style from any of Sony Pictures Animation's previous films" [8, p. 14].Cloudy with A Chance of Meatballs[9] is another film created by SPA that feature a highly stylised approach to character motion, however, it is significantly different to Hotel Transylvania.

According to animators working on the film, Cloudy with A Chance of Meatballs has a style that is broad and customised to the most appropriate choice for a particular shot [10]. This left animators to experiment with the movement style by working collaboratively with the directors to create a complex tapestry of movement patterns. The production of Hotel Transylvania on the other hand was closely scrutinised by its director, GenndyTartakovsky, to achieve a specific and consistent style.

Tartakovsky describes Hotel Transylvania as being inspired by old WB cartoons, specifically referencing the work of animator Frederick Bean (Tex) Avery (1908-1980):

I wanted to bring in more of a Tex Avery, Warner Bros sensibility. I knew it was going to be a little bit of an uphill climb in this day and age because people have forgotten what was so great about that style of animation. I'm not even talking about the big bulging eyeballs or the big crazy takes. It's more about the energy, the performance and the posing.[11] 
International Journal of Computer Graphics \& Animation (IJCGA) Vol.9, No.4, October 2019

The "Tex Avery and Warner Bros" sensibility that Tartakovsky refers to is well known amongst the animation community. Cavalier [12, p. 122] credits Avery and his team with reinventing animation, explaining that they took what was known in character animation and pushed it to the limits with "zany, exaggerated, and extreme style" [12, p. 122]. The image below demonstrates the extreme posing that Tex Avery helped to pioneer:

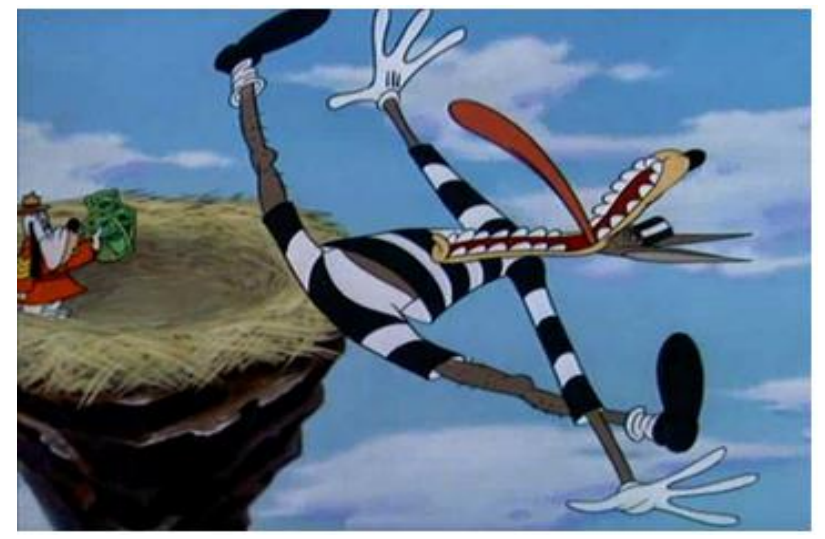

Figure 3. Exaggerated style of Tex Avery

The extreme distortion, sweeping lines and fluid poses evident in Tex Avery's work can be challenging to replicate in 3D animation. The very nature of hand drawn animation supports flexibility in the shapes an animator can create. This is not the case in 3D CG animation, which relies upon complex controls, or "rig", to position an articulated virtual puppet. This technological limitation meant animators had to break the character rigs in Hotel Transylvania to achieve the shapes that Tartakovsky hoped to achieve with the character poses. The technical team had to create new production workflows and approaches to rig deformation to allow the animators to respond to Tartakovsky's direction [13].

Although Tartakovsky did not animate the film himself, (a team of 3D animators did) his influence is seen throughout the film as a result of the production process, which allowed him to critique the animator's work by drawing directly over the top of the unfinished scenes. The following images show Tartakovsky's suggested pose design drawn over the top of the animator's unfinished scene:

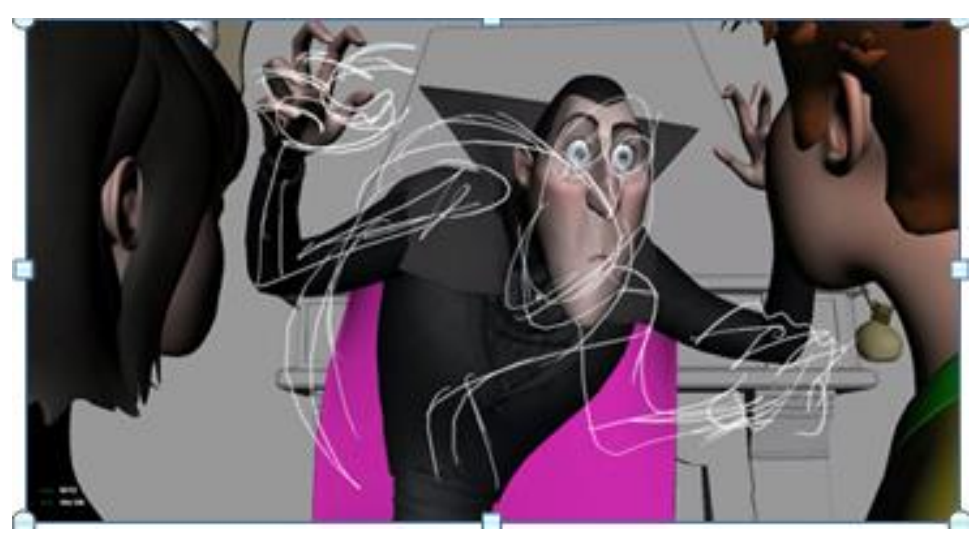

Figure 4.Genndy's suggested pose design [13]. 
International Journal of Computer Graphics \& Animation (IJCGA) Vol.9, No.4, October 2019

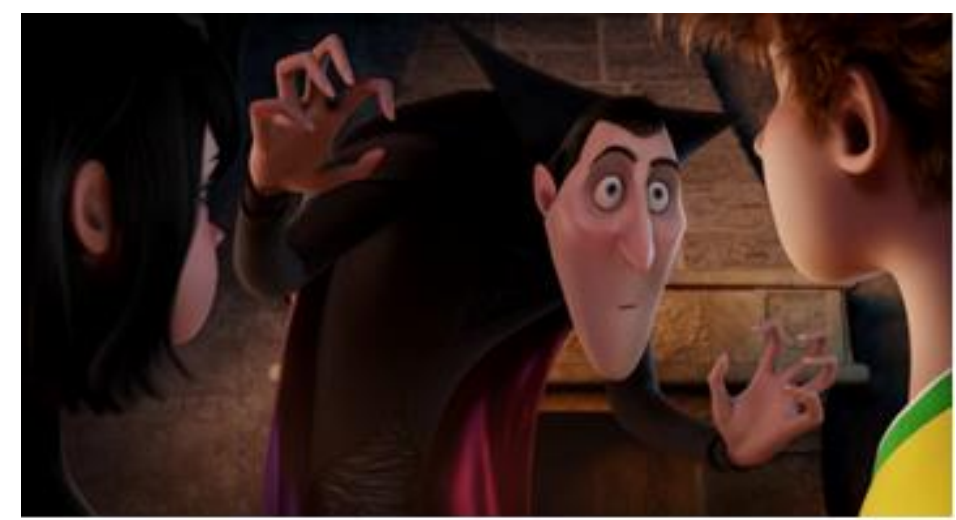

Figure 5. Final rendered frame reflects Genndy's notes [13]

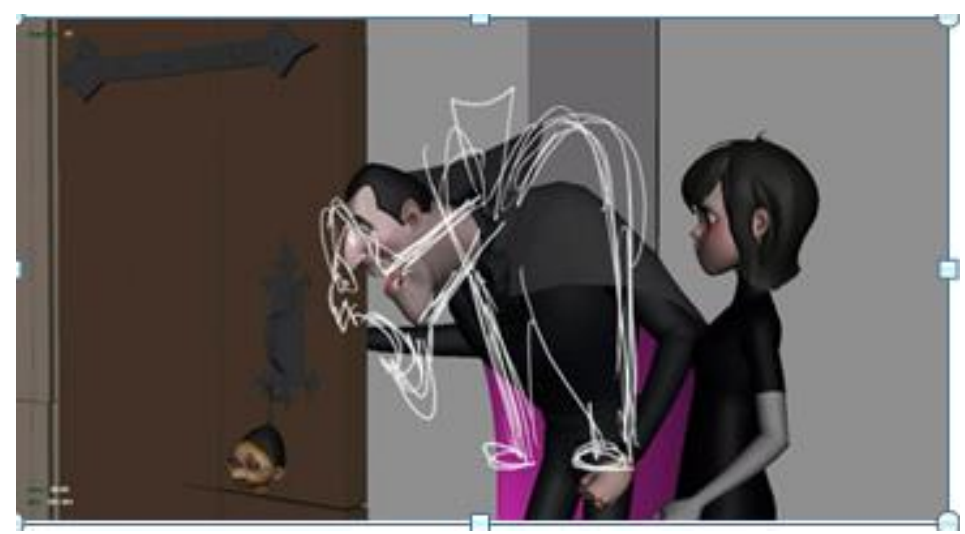

Figure 6.Genndy's suggested pose design [13].

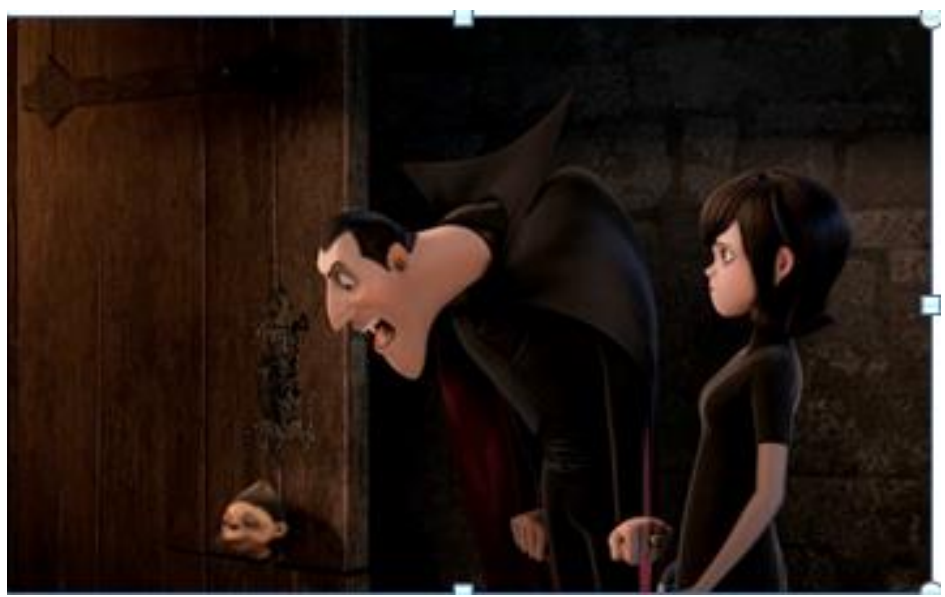

Figure 7. Final rendered frame reflects Genndy's notes [13].

The images above show how Tartakovsky was able to direct the animators to achieve more extreme shapes that are closer to the Tex Avery style he was hoping to achieve. The process of drawing over the top of 3D CG scenes has proven to be an effective way for directors to expand the depth of styles being used in 3D CG animated films. In 2004, director Brad Bird inspired the development of a proprietary tool at PIXAR during production of The Incredibles[14], which was PIXAR's first film to feature a human cast in stylised poses [15]. More recently at Disney, traditional animator Glen Keane worked very closely with the CG animators on Tangled to create 
International Journal of Computer Graphics \& Animation (IJCGA) Vol.9, No.4, October 2019

character poses and motion that extended the traditional Disney style in the 3D CG medium. Directing the film in this way allows the authorial intentions of the director to permeate the entire production by providing animators with new creative ideas and also challenging the technologies that make those ideas possible.

\section{ANALYSIS}

\subsection{Pushed Character Poses}

Perhaps the most obvious stylistic difference between Hotel Transylvania and a Disney style film such as Tangled and Frozen [16]or even a PIXAR film such as Brave [17]or Toy Story 3 [18], is the practice of exaggerating poses by distorting characters into shapes that emphasise their emotional state. Disney and PIXAR films have a tendency to use expressive poses that stretch and deform during moments of broad action and then settle into a pose that does not hyperextend or completely reshape limbs and other elements of the body. In contrast to this, the poses in Hotel Transylvania are often pushed into appealing shapes that force the characters body beyond the limits the audience would expect from a physical being. According to Tartakovsky, this approach allows for a wider range of expressions, ultimately resulting in improved character believability:

To me, it was always ridiculous that you can't emote if you're doing something cartoony and exaggerated. I always argued the opposite. The more cartoony and exaggerated you are, the more range of expression you have and it will be more believable. And so, that was the whole point. Push the expressions. Push the animation. Push the posing to a much more exaggerated level. [19]

Walt Disney also believed exaggeration to be significant to creating effective animation. In 1935, long before Frank Thomas and Ollie Johnston listed exaggeration as a fundamental principle of animation in their text, The Illusion of Life Disney[1], Disney had listed exaggeration as the third most important skill of the top animators. He described exaggeration as being the "ability to caricature action - to take a natural human action and see the exaggerated funny side of it" [20, p. 3]. Thomas and Johnston [1] expanded on this notion when they listed exaggeration as a fundamental principle of animation.

They explain that it created some confusion amongst animators at the studio when Disney asked for more realism only to then be criticised for a lack of exaggeration in the character action. In contrast, when animators created more "distorted drawings or action so violent it was disturbing. They found they had missed the point" [1, p. 65]. Disney was not after realism, rather he was wanting a caricature of realism that would have a bigger impact on the viewer. The result is a style that does not distort the figure but pushes the actions and posture of characters beyond realism to accent the character's emotion.

Although exaggeration is present in both Disney style motion and the motion in Hotel Transylvania, it is approached quite differently. Hotel Transylvania deliberately takes the "distorted drawings" approach by positioning the figure in exaggerated postures that break the physical limitations of the body. This can be seen in the images below, which compare two dynamic poses from Disney's Frozen with several from Hotel Transylvania: 
International Journal of Computer Graphics \& Animation (IJCGA) Vol.9, No.4, October 2019

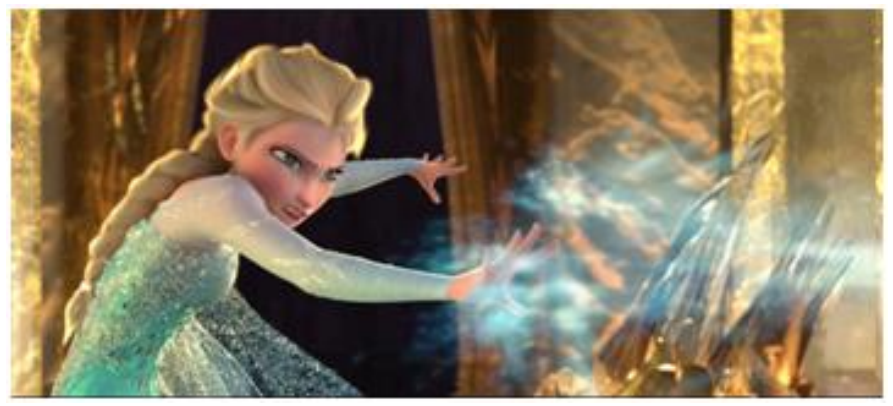

Figure 8. Dynamic pose in Frozen.

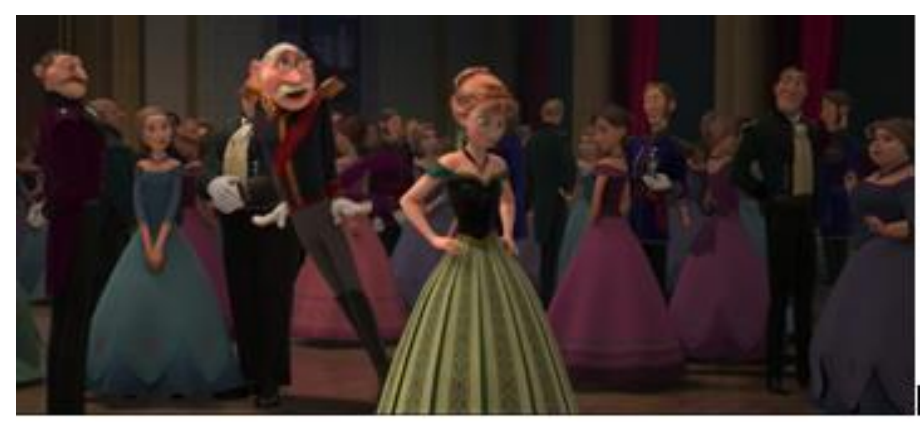

Figure 9. Stretched pose in Frozen.

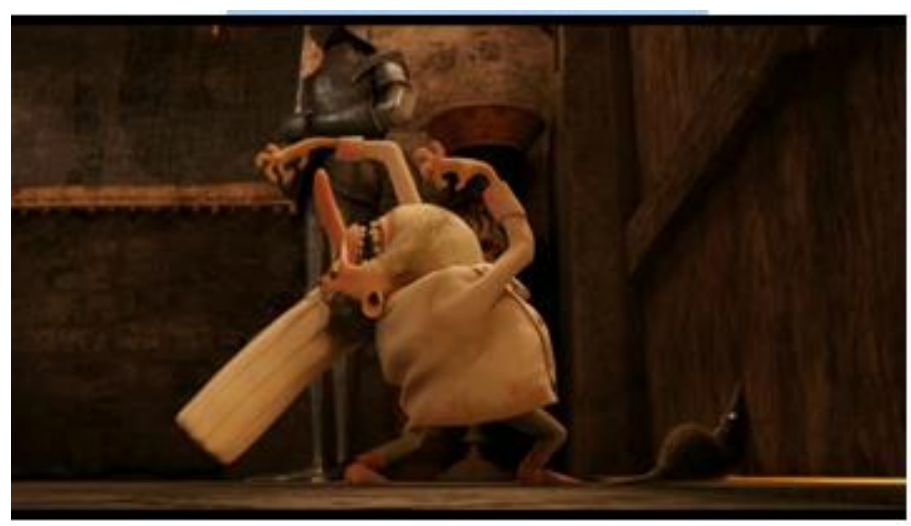

Figure 10. Pushed pose in Hotel Transylvania.

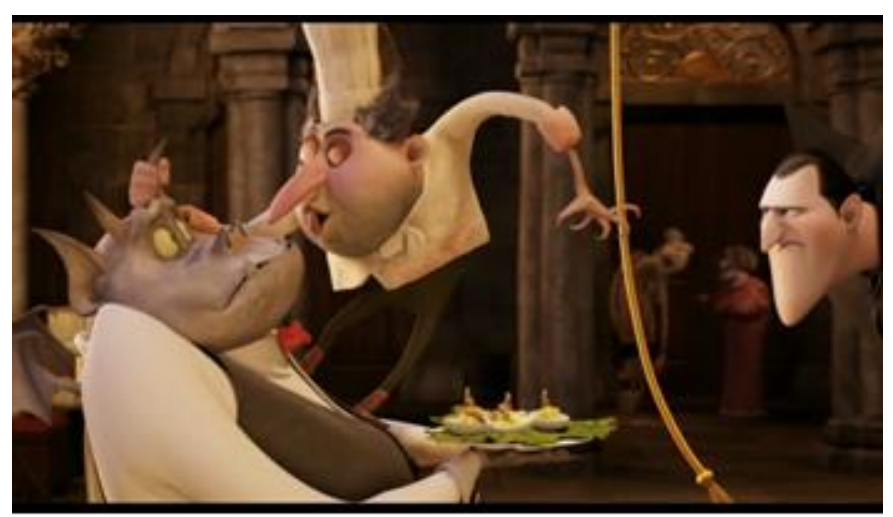

Figure 11. Pushed pose in Hotel Transylvania. 
International Journal of Computer Graphics \& Animation (IJCGA) Vol.9, No.4, October 2019

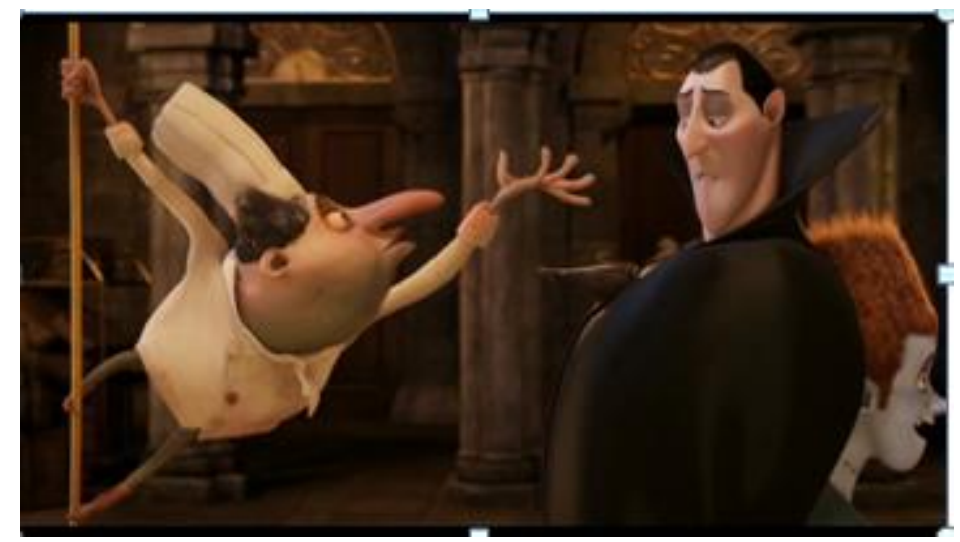

Figure 12. Pushed pose in Hotel Transylvania

The examples shown from Frozen are visually appealing, dynamic and expressive, however, in comparison to Hotel Transylvania they appear to remain very much constrained to the physical limitations of the body. Even in the most comical moments, such as that shown in the second example, the character is not stretched far beyond normal physical limitations. In Hotel Transylvania the posture is exaggerated to emphasise the emotional state of the character with limbs bent and fingers contorted to create interesting shapes far beyond their physical limits.

The images above are not isolated examples from the films; close analysis reveals that this approach to exaggeration is carried throughout. In both films, moments of broad cartoon action are significantly more exaggerated than dramatic emotional moments, which tend to remain visually subdued and in harmony with the intension of the scene. Tartakovsky explains:

People were afraid of holding something for a long time. But it feels great because it gives you that contrast. We have a lot of that quick zippy stuff, but when we do some more dramatic emotional scenes, the characters are very controlled. There is nothing zippy about it.[11]

This approach to blending controlled, subdued motion for dramatic scenes with broad cartoon motion was also used in Disney's Tangled. It not only provides contrast in the film but also promotes authenticity in the character's action, as they are more likely to reflect the emotional context of the scene [6]. Tartakovsky's comments about "quick zippy stuff" highlights that the cartoon stylisation goes beyond the character pose alone. Hotel Transylvania uses fast and drastic changes in shape to create transitions between poses that contribute to the overall exaggerated cartoon stylisation. Again, unlike the Disney approach which tends to rely heavily up the animation principle of squash and stretch, Hotel Transylvania exaggerates the entire shape of the character using a "smear" frame, which is another technique pioneered in 2D cel-based animation to achieve fast appealing motion.

\subsection{Achieving Fast Motion - Smear}

As previously mentioned, Tartakovsky's animation style has been heavily influenced by the early animation that made studios such as WB and MGM famous. An animation "smear" is one technique that developed out of the early experimental works at these studios. The "smear" is typically an elongated drawing that supports the transitioning a character quickly from one pose to another. According to animator Richard Williams [21], the elongated in-between was developed by animators after studying the transparent blurs in live action film. The image below shows Williams' example of what he calls a "zip turn"[21, p. 96]: 

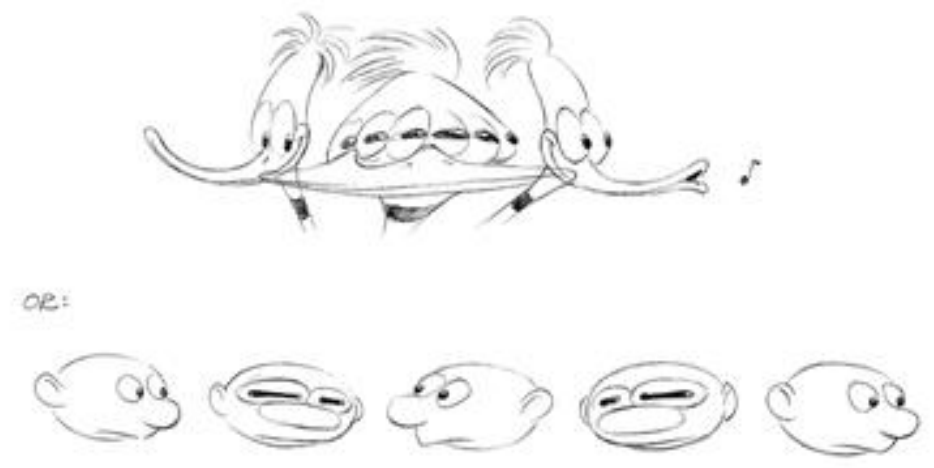

Figure 13. Richard Williams "Zip Turn” using a smear [21, p. 96].

One of the earliest examples of a prolific use of animation smears can be found in the short film directed by Chuck Jones at WB called The Dover Boys at Pimento University[22]. The images below show the villain from the Dover Boys, Dan Backslide, elongated as he moves quickly from one pose to another:

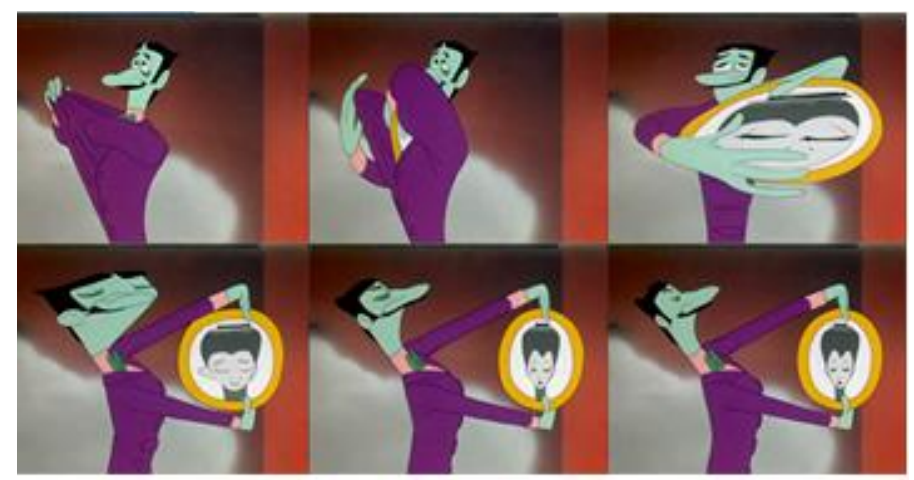

Figure 14. A smear from The Dover Boys at Pimento University.

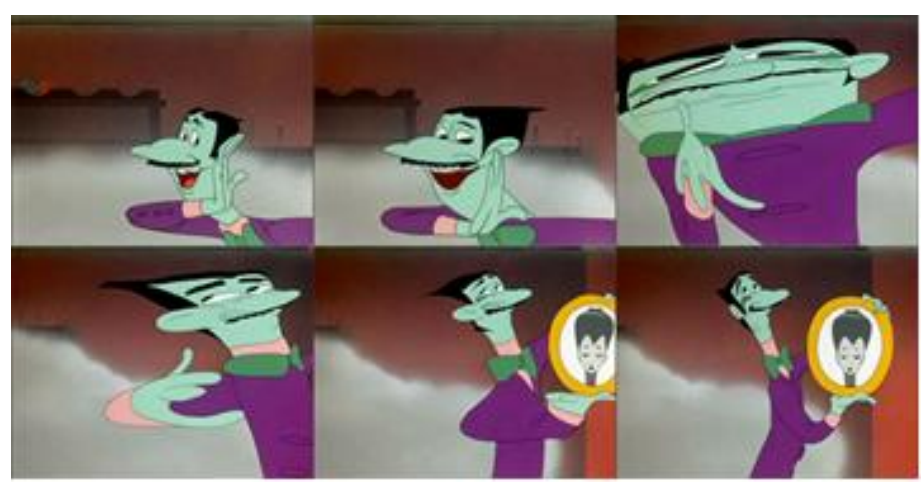

Figure 15. A smear from The Dover Boys at Pimento University.

As these sequences above demonstrate, the character can be wildly distorted into interesting shapes during a smear. In his analysis of the Dover Boys, John Kricfalusi, points out that smear frames are a "cool trick" that is wasted "if you don't have great poses to get to" [2]. Hotel Transylvania demonstrates a well-balanced combination of strong interesting poses and fast expressive smears, the sequences below demonstrate: 
International Journal of Computer Graphics \& Animation (IJCGA) Vol.9, No.4, October 2019

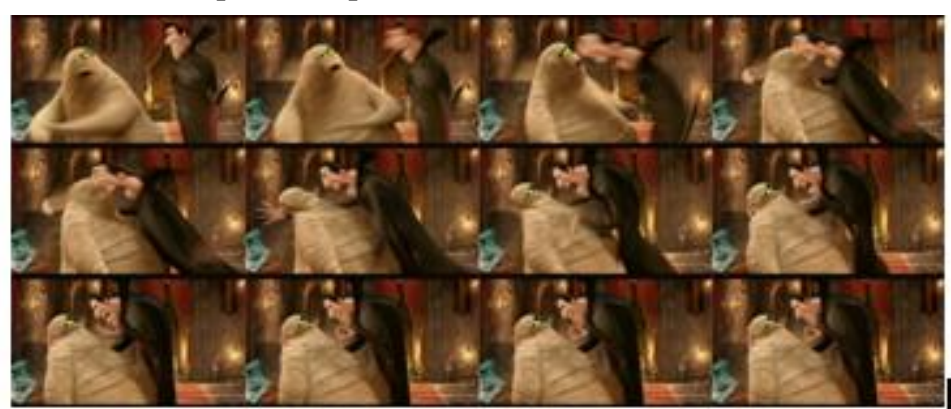

Figure 16. A smear from Hotel Transylvania.

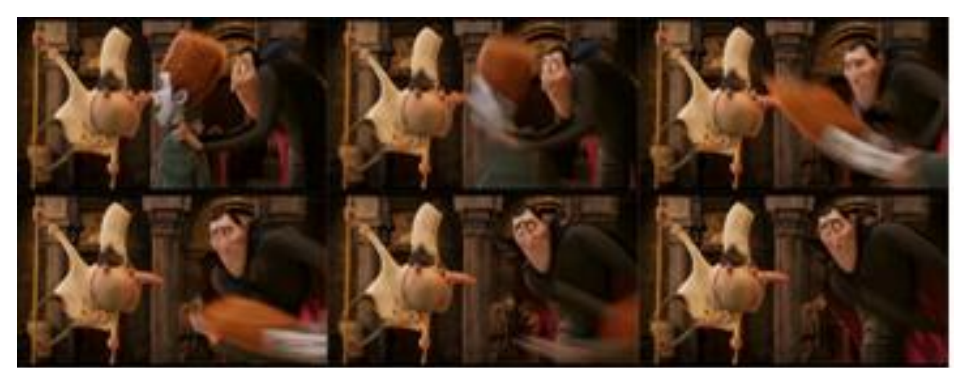

Figure 17. A smear from Hotel Transylvania.

As can be seen above, the character is typically held in an interesting pose with a strong silhouette that easily is read by the audience before being stretched into the next expressive pose over a very short number of frames. This approach to fast action is widespread throughout the film, however, it is not the only technique used.

When beginning this analysis, it was anticipated that the stylisation in Hotel Transylvania would also draw up on a common technique in cel-animation that uses duplicated body parts to sustain the illusion of extreme speed. This technique is known as a "multiple" and can be seen in the two images below, which feature multiple legs during a fast running action:

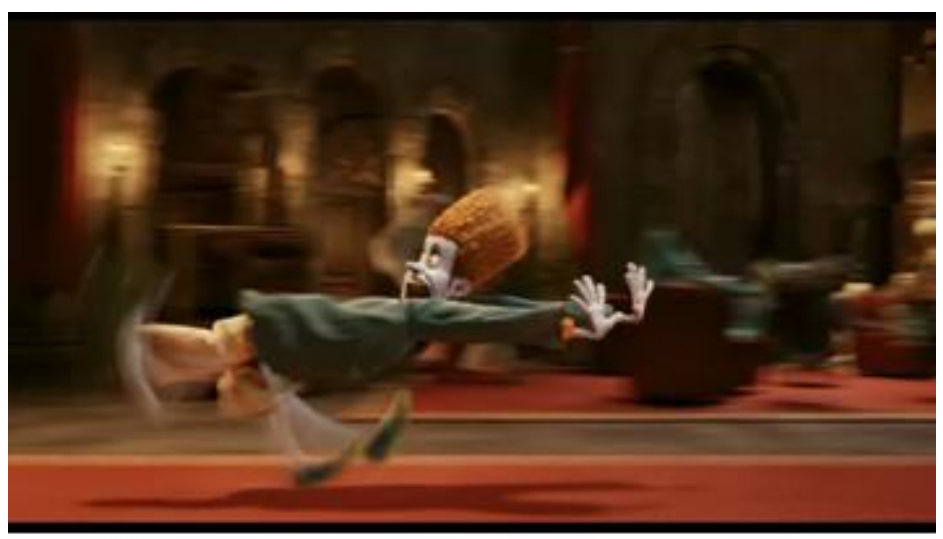

Figure 18. Multiple legs used to create the illusion of fast motion. 
International Journal of Computer Graphics \& Animation (IJCGA) Vol.9, No.4, October 2019

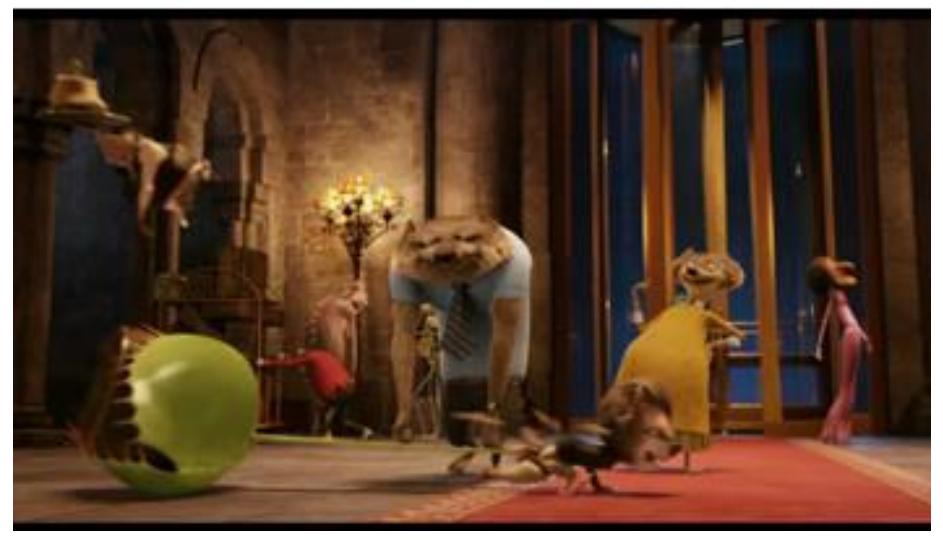

Figure 19. Multiple legs used to create the illusion of fast motion.

Surprisingly, in the scenes and character actions analysed for this study, the two examples above are the only ones in which the use of multiples were used on characters. This indicates multiples may have been reserved for repetitive actions, such as a run cycles, while smear frames were used between exaggerated character poses. As the images above have shown, both techniques are present in the film and blended with a 3D motion blur effect. According to the production notes, the mathematically calculated motion blur of 3D animation had a tendency to reduce the visual impact of these techniques by blurring the interesting shapes that were being created in smear frames. The technical team had to devise a method they dubbed "Genndy Blur" to retain the forms and readability of the character's shape during fast actions [8].

\subsection{Handling Motion Blur - "Genndy Blur"}

Visible blurring or "motion streaks" along the trajectory of fast moving objects is a natural aspect of human perception [23]. This effect is replicated on film when an object moves during the capture process. A longer exposure time for each frame or an increase in an objects speed will result in more visible blurring in the captured image. Audiences are therefore naturally accustomed to experiencing motion blur in objects they see in their environment and on screen. In 3D animation there is no pro-flimic event to record, therefore, motion blur does not naturally occur and must be simulated within the computer. Motion blur is important to 3D animation because it contributes to at least two important aspects of the final imagery seen on screen; it reduces a possible strobing effect for fast moving objects by visually connecting images across frames and simulates natural human perception.

Although necessary to creating appealing 3D animation, motion blur caused some problems for the visual style Tartakovsky was trying to achieve in Hotel Transylvania because it reduced the visual impact of the character poses, he explains:

That whole issue of motion blur was basically my enemy because it blurs all these great drawings away. So, we had to find a system that retained some of the fun and energy of the animation without it blurring away to nothing. Not to get too technical, and I didn't even know this, but the animators had sub-frames in between frames. So, we'd go in and add extra poses in the sub-frames and that tempered the motion blur a little bit.[11]

This idea of "tempering" the motion blur was aimed at reducing the transparency and streaking of the images when characters move very quickly. In the filmmaker's commentary on the DVD release, Tartakovsky expands upon the technique when he mentions that multiple passes (renders) were created and then blended together to create clarity in the poses. In a short documentary explaining the technique, Sony Pictures Animation describe their approach to handling the motion 
International Journal of Computer Graphics \& Animation (IJCGA) Vol.9, No.4, October 2019

blur as "Genndy Blur" due to the use of "additional key frames between poses, frame specific motion blur and a lot of artistic blending" [24]. The image below features Dracula doing a front flip during a dance scene before and after motion blur has been applied:

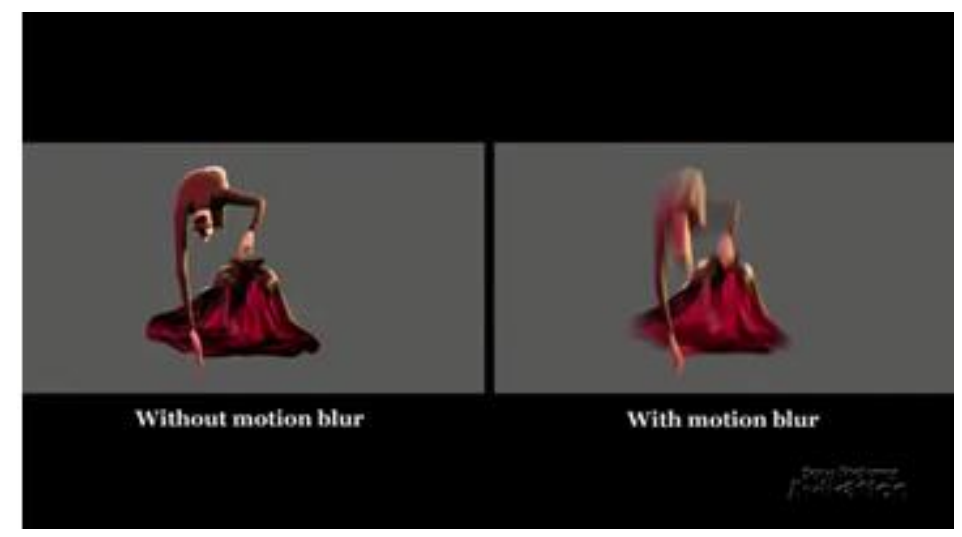

Figure 20. Before and after motion blur[24].

The application of motion blur clearly decreases the clarity of the character shape as the image becomes transparent while streaked in the direction of travel. The image below offers a side-byside comparison of standard motion blur and the improved result of using additional poses to reduce the effect of motion blur:

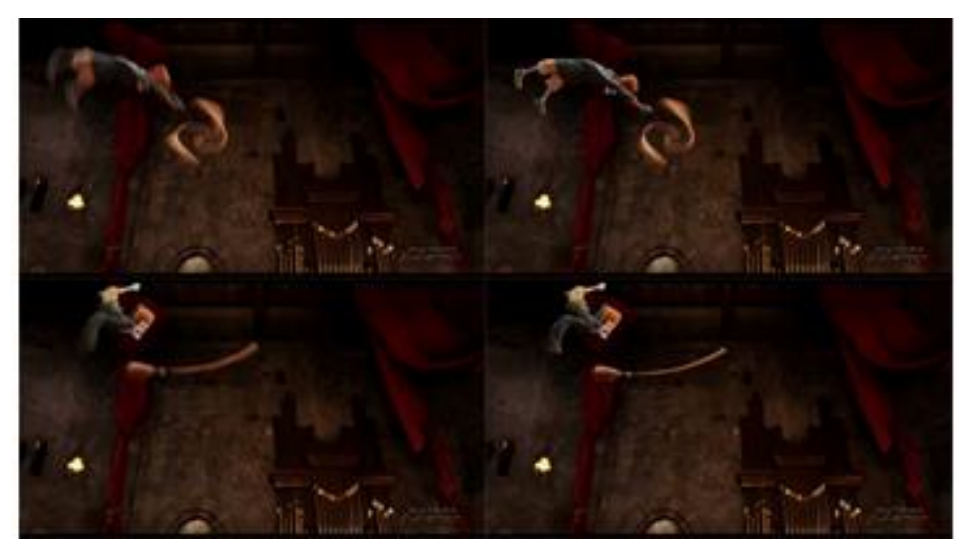

Figure 21. Before and after Genndy Blur [24].

These two examples clearly demonstrate that more clarity was achieved by "tempering" the motion blur. Two brooms are also used in the top two images; this is another example of using multiples (although not on a character) for creating high-speed cyclic motion.

The image below shows an example of an elongated pose blended with motion blur in such a way that it retains the facial features and shape of Dracula's head but allows the body to blur. 
International Journal of Computer Graphics \& Animation (IJCGA) Vol.9, No.4, October 2019

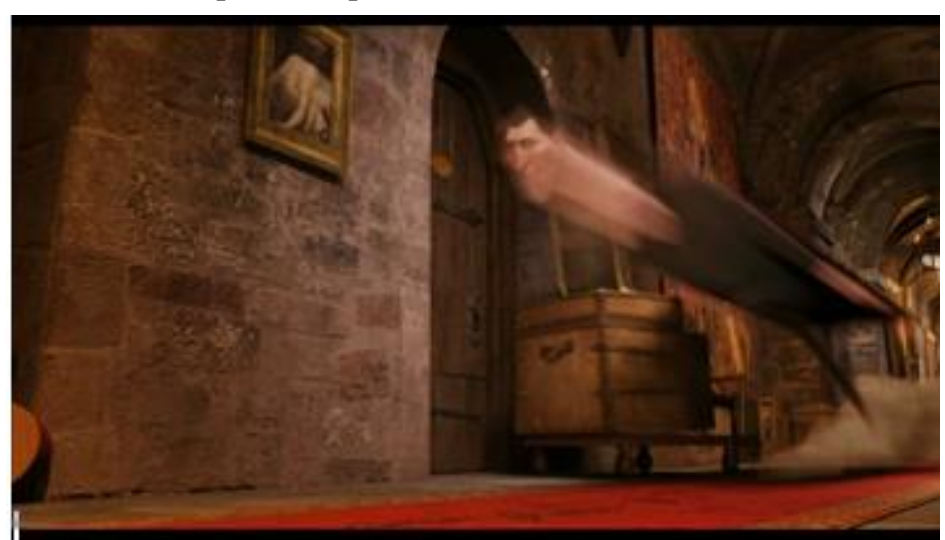

Figure 22. Tempered motion blur in Hotel Transylvania.

In the filmmaker's commentary, visual effects supervisor, Daniel Kramer explains that along with doing multiple passes with varying amounts of motion blur they would also "bias the motion blur toward the front of the character line" to retain the silhouettes that Tartakovsky wanted to achieve. Overall the approach allows for the successful combination of extremely pushed poses and very fast motion in a $3 \mathrm{D}$ animated film.

\section{Conclusions}

Animators such as John Lasseter once argued that traditional animation principles developed at the Disney studio could and should be applied to the 3D CG medium [25], [26]. Since then, traditional animation principles have successfully been adapted and applied to creating believable 3D animated characters and creatures. This aesthetic progression has very much focussed on extending the naturalistic Disney style to the new medium. In cel-based animation, however, the principles of animation were not always used to create naturalistic movement, other studios such as WB, MGM, and UPA pushed the limits of animation and created a diversity of movement qualities that often operated outside the limitations of the physical world. The range of movement styles in the 3D CG medium has continued to broaden as animators experienced in these other forms of animation, such as GenndyTartakovsky, bring with them a desire to create 3D animation that reflects their own animation sensibilities.

As this study has shown, Hotel Transylvania in an excellent example of how the diverse movement styles that developed in cel-based animation have begun to expand the 3D CG medium beyond the dominant naturalist style of Disney and PIXAR animation. GenndyTartakovsky's exaggerated animation sensibilities were able to shape the movement style of the film because of a production process that allowed Tartakovsky to literally draw over the top of the animation created by $3 \mathrm{D}$ animators. This approach to critiquing their work, helped guide the $3 \mathrm{D}$ animators toward creating the shapes and postures that Tartakovsky wanted to see in the film.

Hotel Transylvania uses exaggerated character poses inspired by old WB cartoons and animation pioneers such as Tex Avery and Chuck Jones. There is a deliberate focus on creating interesting shapes and fast staccato movement rather than physically accurate postures and motion. This is combined with smear frames that allow characters to move quickly between extremes positions while retaining a certain visual clarity. Frame specific motion blur has been combined with additional key frames and extreme poses to ensure the interesting shapes created during fast movements would retain their silhouettes. Not all actions are exaggerated and fast; Tartakovsky has created contrast in the movement vocabulary by combining moments of extreme cartoon exaggeration with very controlled and subdued movement for dramatic moments. Tartakovsky and 
International Journal of Computer Graphics \& Animation (IJCGA) Vol.9, No.4, October 2019

the animation team that worked on Hotel Transylvania havesuccessfully extended the exaggerated cartoon style of studios such as WB and MGM into the 3D CG medium.

\section{REFERENCES}

[1] F. Thomas and O. Johnston, The illusion of life: disney animation. New York, NY, USA: Disney Editions, 1995.

[2] J. Kricfalusi, “John K Stuff: Smears and Poses,” John K Stuff, 09-Jul-2009.

[3] T. G. Hu, Frames of anime: culture and image-building. Hong Kong University Press, 2010.

[4] G. Tartakovsky, Hotel Transylvania. 2012.

[5] N. Greno and B. Howard, Tangled. Walt Disney Studios, 2010.

[6] C. P. Carter, "An Analysis of the Character Animation in Disney's Tangled," Senses Cine., no. 67, 2013.

[7] IMDb, "GenndyTartakovsky," IMDb. [Online]. Available: http://www.imdb.com/name/nm0850733/. [Accessed: 18-May-2014].

[8] Sony Pictures Animation, "Hotel Transylvania Production Notes.” Visual Hollywood, 2012.

[9] P. Lord and C. Miller, Cloudy with a Chance of Meatballs. 2009.

[10] L. Vasquez, "Interview With Cloudy With a Chance of Meatballs 2 Animators," 2013.

[11] G. Tartakovsky, “GenndyTartakovsky Talks ‘Hotel Transylvania,”” 27-Sep-2012.

[12] S. Cavalier, The World History of Animation. University of California Press, 2011.

[13] B. Desowitz, “'Hotel Transylvania' Gets a 2D Transfusion,” Animation World Network, 27-Sep-2012.

[14] B. Bird, TheIncredibles. Disney Pixar, 2004.

[15] E. Wolff, "Tool time at pixar: sketching the incredibles," 01-Nov-2004.

[16] C. Buck and J. Lee, Frozen. Walt Disney Animation Studios, 2013.

[17] M. Andrews, B. Chapman, and S. Purcell, Brave. Walt Disney Pictures, 2012.

[18] L. Unkrich, Toy Story 3. Walt Disney Pictures, 2010.

[19] G. Tartakovsky, "Hotel Transylvania Director GenndyTartakovsky Uses Looney Tunes Logic To Push Animation Envelope," 28-Sep-2012.

[20] W. Disney, "Walt disney productions inter office communication," 1935.

[21] R. Williams, The animator's survival kit-revised edition: a manual of methods, principles and formulas for classical, computer, games, stop motion and internet animators. Faber \& Faber, Inc., 2009.

[22] C. M. Jones, The Dover Boys at Pimento University or The Rivals of Roquefort Hall. 1942. 
International Journal of Computer Graphics \& Animation (IJCGA) Vol.9, No.4, October 2019

[23] D. Apthorp, D. S. Schwarzkopf, C. Kaul, B. Bahrami, D. Alais, and G. Rees, "Direct evidence for encoding of motion streaks in human visual cortex," Proc. R. Soc. B Biol. Sci., vol. 280, no. 1752, pp. 20122339-20122339, Dec. 2012.

[24] Sony Pictures Animation, Hotel Transylvania - Genndy Blur Shot Build. 2013.

[25] J. Lasseter, "Principles of traditional animation applied to 3D computer animation," ACM SIGGRAPH Comput. Graph., vol. 21, no. 4, pp. 35-44, 1987.

[26] J. Lasseter, "Tricks to animating characters with a computer," ACM SIGGRAPH Comput. Graph., vol. 35 , no. 2, pp. 45-47, 2001.

\section{Author}

Dr Chris Carter is a Senior Lecturer in Animation in the Creative Industries Faculty at Queensland University of Technology. His research focus is on animation aesthetics and animated miseen scène. 